\title{
Efficient searching for grain storage container by combine robot
}

\section{AUTHOR(S):}

Kurita, Hiroki; lida, Michihisa; Suguri, Masahiko; Masuda, Ryohei; Cho, Wonjae

\section{CITATION:}

Kurita, Hiroki ...[et al]. Efficient searching for grain storage container by combine robot. Engineering in Agriculture, Environment and Food 2014, 7(3): 109-114

\section{ISSUE DATE:}

2014-07

URL:

http://hdl.handle.net/2433/189412

\section{RIGHT:}

(C) 2014 Asian Agricultural and Biological Engineering Association. Published by Elsevier B.V.; この論文は出版社版でありません。引用の際には出版社版をご確認ご利用ください 。; This is not the published version. Please cite only the published version. 


\title{
Efficient Searching for Grain Storage Container by Combine Robot ${ }^{*}$
}

\author{
Hiroki KURITA $^{* 1}$, Michihisa IIDA ${ }^{* 2}$, Masahiko SUGURI ${ }^{* 3}$, Ryohei MASUDA ${ }^{* 3}$, Wonjae CHO*1
}

\begin{abstract}
In this study, a combine robot was equipped with an autonomous grain container searching function. In order to realize automated grain unloading, the combine robot has to search and identify the grain storage container in an outdoor environment. A planar board was attached to the container. The marker was searched for using a camera mounted on the unloading auger of the combine. An efficient marker searching procedure was proposed on the basis of a numerical analysis of the camera's field of view and was verified experimentally. The results showed that the combine robot efficiently searched for and detected the marker and positioned its spout at the target point over the container to unload the grain.
\end{abstract}

[Keywords] head-feeding combine robot, grain unloading operation, grain container searching operation, spout positioning, field of view

\section{Introduction}

In Japan, the number of workers engaged in agriculture is decreasing, and the average age of agricultural workers is rapidly increasing. Food self-sufficiency in Japan remains low compared to other developed countries. Japan must improve its agricultural productivity in order to maintain its sustainability. Field robots are expected to play an important role in improving the efficiency of agricultural operations and meeting workforce shortages. Attempts to develop automated agricultural machinery have previously been reported (Noguchi and Terao, 1997; Ishida et al., 1998; Nagasaka et al., 2004; Takai et al., 2010). In a previous study (Iida et al., 2012), we robotized a head-feeding combine harvester (hereafter referred to as a combine) and used it to harvest rice and wheat in fields. The combine robot successfully traveled along a target path and harvested rice crops autonomously.

However, a human operator is needed to manually control the combine and unload grain from its grain tank into a separate grain storage container. We aimed to automate the unloading operation as well. A pickup truck is driven and parked by a human driver on a farm road. The parking position of the truck is determined in advance. As the combine robot can obtain this parking position as Global Positioning Satellite (GPS) data, it

27 autonomously travels to a position near the truck when 28 the grain tank is full. However, the position of the 29 combine relative to the pickup truck is not strictly fixed, 30 because the human driver cannot perfectly park the 31 pickup truck without positional errors. Thus, the 32 combine robot has to find the pickup truck by an image 33 processing technique and then correct its relative 34 position to unload grain into the truck without any loss.

35 Kurita et al. (2012) utilized an image processing 36 technique to appropriately position the unloading auger 37 to unload grain. Figure 1 shows the assumed situation 38 for their concept.

39

40

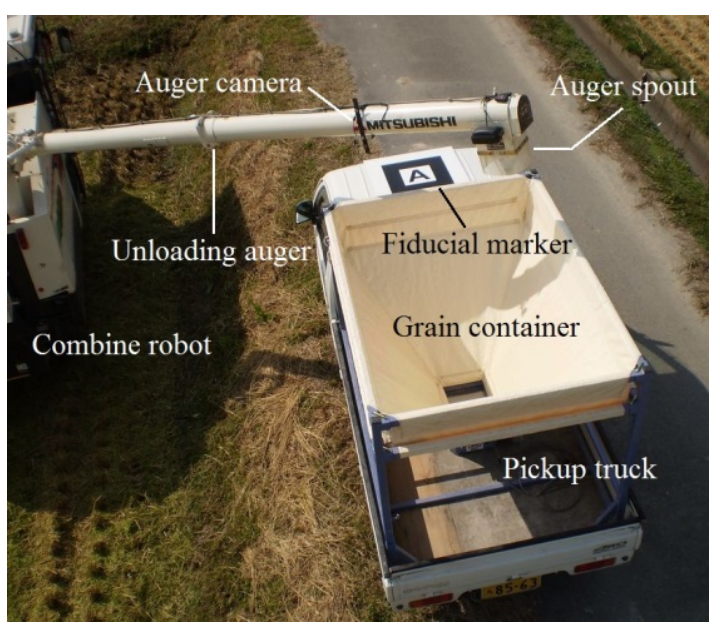

Fig. 1. Setup of autonomous unloading operation.

* Partly presented at the $6^{\text {th }}$ International Symposium on Machinery and Mechatronics for Agricultural and Biosystems Engineering ISMAB 2012

*1 JSAM Student Member, Graduate School of Agriculture, Kyoto University, Kitashirakawa Oiwake-cho, Sakyo-ku, Kyoto, 606-8502, Japan

*2 JSAM Member, Corresponding author, Graduate School of Agriculture, Kyoto University, Kitashirakawa Oiwake-cho, Sakyo-ku, Kyoto, 606-8502, Japan ; iida@elam.kais.kyoto-u.ac.jp

*3 JSAM Member, Graduate School of Agriculture, Kyoto University, Kitashirakawa Oiwake-cho, Sakyo-ku, Kyoto, 606-8502, Japan 

$400 \mathrm{~mm}$ ) is placed on the roof of the pickup truck to detect the position of the grain container. The position is 4 extracted from images captured by the camera attached 5 to the unloading auger. On the basis of the extracted 6 image features, the positional relation between the 7 combine and container is determined using image 8 processing techniques. The experimental results showed 9 that the auger spout can be visually positioned at the the FOV for any parameter with ease. Thus, the objectives of this study were as follows: to compute the FOV of the auger camera against FOV parameters based on the pinhole camera model, propose a marker searching algorithm in order to efficiently search for and accurately detect the marker and examine the actual performance of the proposed method with a combine robot.

\section{Materials and Methods}

1. Kinematic Modeling and Mechanics of Unloading Auger

The test vehicle was a head-feeding combine harvester, VY50 CLAM (Mitsubishi Agricultural Machinery Co., Ltd, Shimane, Japan). The unloading auger of the combine was modeled with a two-degrees-of-freedom manipulator consisting of two joints (joints 1 and 2). As illustrated in Fig. 2, a right-handed coordinate system was assigned to the combine; the $x$ axis of the coordinate system was along the body of the combine in the direction opposite to the direction of its motion, and the $z$ axis pointed vertically upward. The state of the unloading auger was determined by the two joint angles (hereafter $\theta_{1}$ and $\theta_{2}$ ). Joint 1 rotated at an angle of $-110^{\circ}$ $<\theta_{1}<200^{\circ}$. The grain could be discharged when $-110^{\circ}$ $<\theta_{1}<90^{\circ}$. However, unloading was expected to be performed when $20^{\circ}<\theta_{1}<90^{\circ}$. Joint 2 rotated at an angle of $0^{\circ}<\theta_{2}<45^{\circ}$.

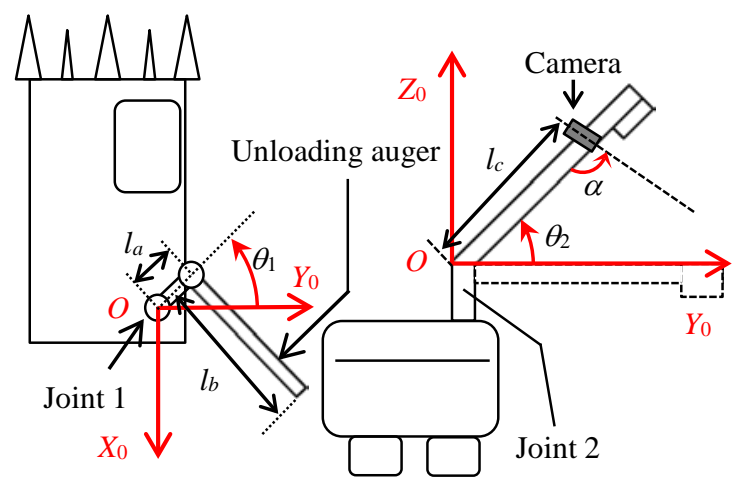

Fig. 2. Kinematic model of unloading auger.

Joints 1 and 2 were actuated by a DC motor and hydraulic cylinder, respectively. Each joint rotated at a constant rate: $38.3 \%$ for joint 1 and $20.7 \%$ s (upward) and $10.7 \%$ (downward) for joint 2 with on-off control. Link lengths $l_{a}, l_{b}$, and $l_{c}$ were defined as shown in Fig. 2. The camera's elevation angle $\alpha$ was set to $71^{\circ}$. Table 1 lists the specifications of the camera.

Table 1. Camera specifications.

\begin{tabular}{cc}
\hline Model & UCAM-DLA200H (ELECOM) \\
Image sensor & $1 / 4$ in CMOS \\
Focal length & $4.3 \mathrm{~mm}$ \\
F-number & 1.8 \\
Angle of view (diagonal) & $60^{\circ}$ \\
\hline
\end{tabular}




\section{FOV of Auger Camera}

A rice paddy is usually enclosed by embankments and at least one farm road (see Fig. 3).

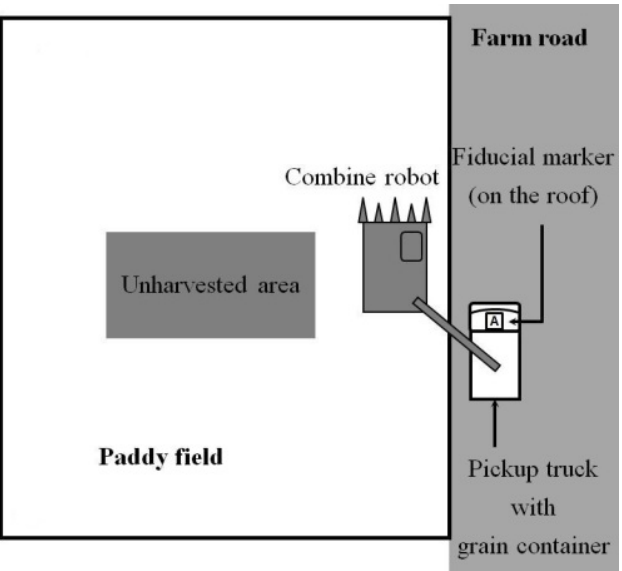

Fig. 3. Positional relation between the combine and the truck.

A commercialized head-feeding combine harvester is

equipped with its unloading auger on the right side of the vehicle (see Fig. 3). As is the case for manned harvesting, the combine robot harvests rice crops in an anticlockwise fashion (Iida et al., 2012). Thus, in this study, the truck was always located on the right side of the combine. Let $h_{f r}$ be the height of the adjacent farm road from a paddy field, $h_{c}$ be the height of joint 1 of the combine harvester, and $h_{k t}$ be the height of the pickup truck.

In general, the FOV can be represented by its angle of view (AOV) and depth of field (DOF). The AOV comprises the vertical and horizontal AOV. The DOF represents the area of the visual scene that is acceptably sharp. Outside of this range, images are blurred. The DOF depends on the focal length of the camera. In this study, the focal length of the camera was kept constant so that the DOF would fall within an acceptable range of sharpness. The DOF was empirically determined; at the same time, the target plane (i.e., the roof of the truck) was experimentally confirmed to form an image with sufficient sharpness for the expected range of the height from the paddy field to the farm road $h_{f r}$. In this study, the range was assumed to be $0 \mathrm{~m}<h_{f r}<1.5 \mathrm{~m}$.

A pinhole camera model (Gonzalez and Wintz, 1987; $\mathrm{Xu}$ and Zhang, 1996) was adopted for the following simulation. Further approximations were applied to the system model. In the following analysis, the unloading auger was dealt with as a line, not a solid object, and the camera's dimensions were neglected. Hence, $l_{a}$ was assumed to be $0.0 \mathrm{~m}$. The values $l_{b}$ and $l_{c}$ were 4.280 and $3.195 \mathrm{~m}$, respectively. It may be considered that $l_{c}$ is also

40 a decisive variable for the FOV. Obviously, the camera 41 needs to be attached at the top of the unloading auger to 42 obtain as large an FOV as possible. When attached to the 43 spout, however, the camera cannot obtain a clear image 44 because of the dust that flows out of the spout during the 45 unloading operation. Thus, the camera was attached as 46 close to the auger spout as possible without being 47 obstructed by dust from the unloading operation. This 48 position was empirically determined and regarded as 49 constant throughout the study.

50 When $h_{c}$ was longer than $h_{k t}+h_{f r}$, the associated 51 parameters were as depicted in Fig. 4, which represents 52 the cross-sectional view at the $Y_{0}-Z_{0}$ plane when $\theta_{1}=$ $5390^{\circ}$.

68

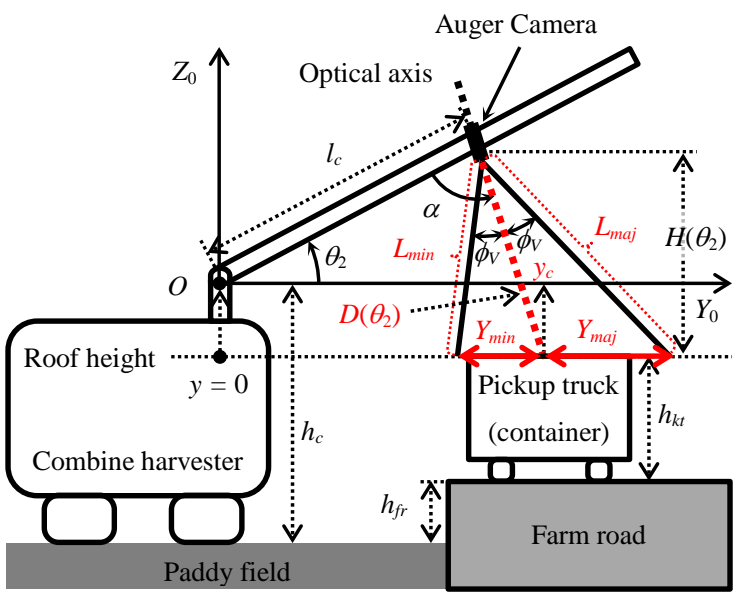

Fig. 4. Cross-sectional view at the $Y_{0}-Z_{0}$ plane.

The value of $\phi_{V}$ is half that of the vertical AOV. The FOV at the height of the fiducial marker on the roof is divided by the optical axis (the point $y_{c}$ in Fig. 4) into two parts; the lengths of these parts along the $Y_{0}$ axis are $Y_{\min }$, which is closer to the combine, and $Y_{m a j}$, which is farther. $L_{\text {min }}$ and $L_{m a j}$ are the distances between the upper or lower edges of the FOV at the roof height and optical center, respectively. The depth of field at the marker plane $D\left(\theta_{2}\right)$ is represented as

$$
D\left(\theta_{2}\right)=\frac{H\left(\theta_{2}\right)}{\sin \left(\theta_{2}+\alpha\right)}
$$

where $H\left(\theta_{2}\right)$ is $l_{c} \sin \theta_{2}+\left(h_{c}-h_{f r}-h_{k t}\right)$. Similarly, $L \min$ and $L_{\text {maj }}$ are obtained as follows:

$$
L_{\text {min }}=\frac{H\left(\theta_{2}\right)}{\sin \left(\theta_{2}+\alpha-\phi_{V}\right)}
$$

$$
L_{m a j}=\frac{H\left(\theta_{2}\right)}{\sin \left(\theta_{2}+\alpha+\phi_{V}\right)}
$$

69 Then, $Y_{\min }$ and $Y_{\operatorname{maj}}$ are written as follows: 


$$
Y_{\text {min }}=\sqrt{\left\{D\left(\theta_{2}\right)\right\}^{2}+L_{\text {min }}^{2}-2 D\left(\theta_{2}\right) L_{\text {min }} \cos \phi_{V}}
$$

1

$$
Y_{m a j}=\sqrt{\left\{D\left(\theta_{2}\right)\right\}^{2}+L_{m a j}^{2}-2 D\left(\theta_{2}\right) L_{m a j} \cos \phi_{V}}
$$

2 The value of $y_{c}$ is obtained geometrically using Eq. (6):

$$
\begin{array}{r}
y_{c}=\sqrt{\left\{D\left(\theta_{2}\right)\right\}^{2}+l_{c}{ }^{2}-2 D\left(\theta_{2}\right) l_{c} \cos \alpha} \\
+\frac{h_{c}-h_{f r}-h_{k t}}{\tan \left(\theta_{2}+\alpha\right)}
\end{array}
$$

Figure 5 shows the FOV and parameters that are also 4 illustrated in Fig. 4. To make the figure clearer, the plane 5 including the line of $L_{\min }$ and the lower edge is clipped 6 and shown separately on the left side. Here, $\phi_{H}$ is half of 7 the horizontal AOV. $X_{\min }$ is half of the bottom length of 8 the rectangular triangle consisting of the angle $\phi_{H}$ and the 9 line segment with the length $L_{\min }$. Hence,

$$
X_{\min }=L_{\min } \tan \phi_{H}
$$

Similarly, the plane that includes the line of $L_{m a j}$ and the upper edge is depicted separately in the right side, and $X_{m a j}$ is defined along with $X_{\min }$.

$$
X_{m a j}=L_{m a j} \tan \phi_{H}
$$

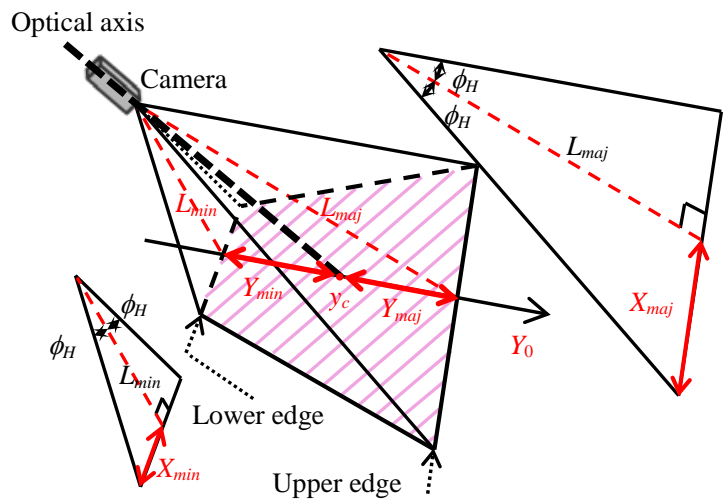

Fig. 5. Conceptual image of FOV.

Finally, the FOV can be simulated using the parameters $y_{c}, X_{\min }, X_{m a j}, Y_{m i n}$, and $Y_{m a j}$. When $h_{c}$ is shorter than $h_{k t}+$ $h_{f r}$, the FOV can be simulated in a manner similar to the case of $h_{c}>h_{k t}+h_{f r}$.

\section{Auger Control for Searching Operation}

Figure 6 shows the maximal FOV for the representative $h_{f r}$ values of $0.0,0.5,1.0$, and $1.5 \mathrm{~m}$ on the combine-based coordinate system (i.e., $\mathrm{O}-\mathrm{X}_{0} \mathrm{Y}_{0} \mathrm{Z}_{0}$ in Fig. 2) when $\theta_{1}$ of the unloading auger rotates from $180^{\circ}$ to $270^{\circ}$. Note that the point $\left(X_{0}, Y_{0}\right)=(0,0)$ in Fig. 6 , which is 28 marked as Origin, indicates the origin of the 29 combine-based coordinate system. Especially for $\theta_{1}=0^{\circ}$, $3090^{\circ}$, and $180^{\circ}$, the FOVs at $\theta_{2}=20^{\circ}, 30^{\circ}, 40^{\circ}$, and $45^{\circ}$ 31 have a trapezoidal shape; among them, the FOV at $20^{\circ}$ is 32 the narrowest and that at $45^{\circ}$ is the widest. In short, each 33 maximal FOV shows the total area captured by the camera when $\theta_{2}$ varies from $20^{\circ}$ to $45^{\circ}$. In Figs. 6(a) and
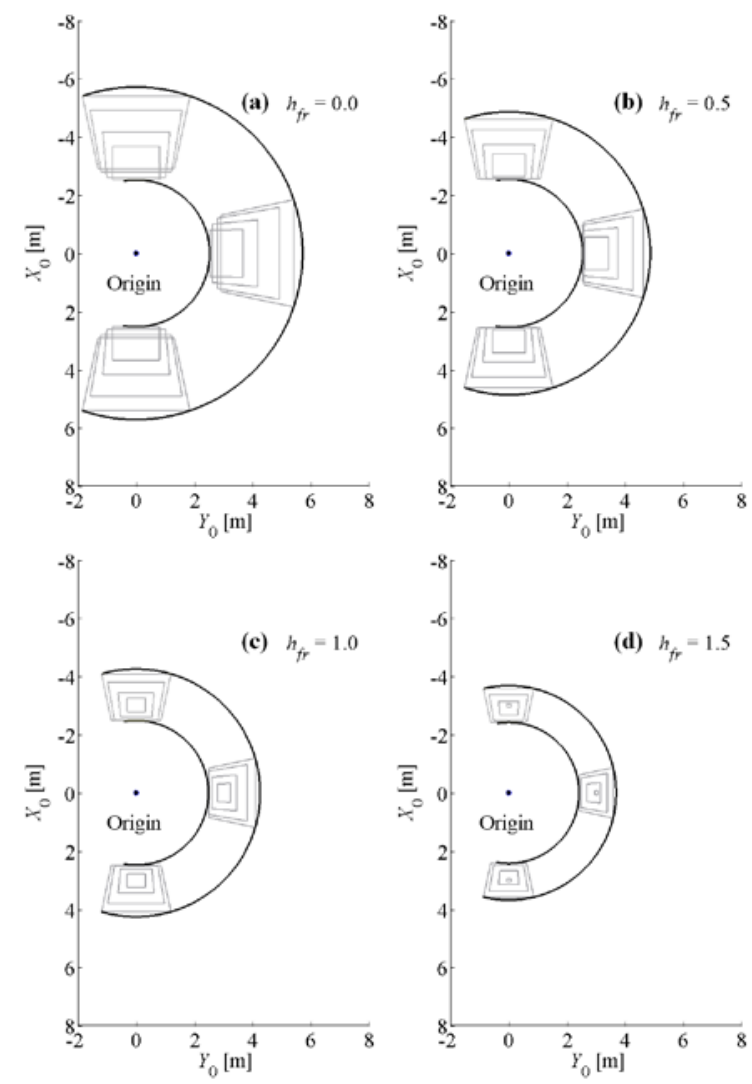

Fig. 6. Maximal FOV.

(a). $h_{f r}=0.0 \mathrm{~m}$; (b). $h_{f r}=0.5 \mathrm{~m}$;

(c). $h_{f r}=1.0 \mathrm{~m}$; (d). $h_{f r}=1.5 \mathrm{~m}$.

However, if the unloading auger rotates with $\theta_{2}=20^{\circ}$, physical interference may arise between the auger and grain storage container, especially when $h_{f r}=1.5 \mathrm{~m}$. 51 Thus, the searching procedure should be performed at $\theta_{2}$ $52=30^{\circ}$ and $\theta_{2}=45^{\circ}$. Because the maximal FOV can be 53 almost entirely covered using only the FOV at $\theta_{2}=45^{\circ}$, 54 the searching procedure should be performed primarily 
1 at $\theta_{2}=45^{\circ}$ and then at $\theta_{2}=30^{\circ}$. Hereinafter, the former

2 is the primary searching step, and the latter is the 3 secondary searching step.

The entire searching procedure is as follows. First, the 5 two joints of the unloading auger are kept at $\theta_{1}=192.0^{\circ}$ and $\theta_{2}=2.6^{\circ}$, which is the state that the combine usually travels and harvests in (let this state be the default 8 position). The auger moves upward to $\theta_{2}=45^{\circ}$ and 9 rotates clockwise to $\theta_{1}=0^{\circ}$ (the primary step). The 10 combine inevitably needs to keep $\theta_{2}=45^{\circ}$ for the 11 rotation from the default position to prevent the unloading auger from crashing into the cab. Then, the auger drops down to $\theta_{2}=30^{\circ}$ and rotates anticlockwise to $\theta_{1}=90^{\circ}$ (the secondary step). If the marker is not detected, the auger returns to the default position. During the search, only the marker detection is performed with the image processing. When the fiducial marker is detected, the auger stops immediately and rests for $2 \mathrm{~s}$ to obtain clearer images, which are used to calculate the precise target joint angles. After that, positioning is performed.

More than $92 \%$ of the maximal FOV can be covered by the primary step; with the additional searching by the secondary step, a coverage of more than $98 \%$ is achieved. Since the unloading auger rotates at a regular rate, the maximum times required for the primary and secondary steps are 7.9 and $4.9 \mathrm{~s}$, respectively. The auger stops for $1 \mathrm{~s}$ to switch the searching mode from the primary step to the secondary step.

\section{Field Experiment}

We conducted an experiment to confirm the viability of the proposed searching-positioning method and to evaluate its efficiency. A combine robot was parked alongside a pickup truck, and a fiducial marker was placed on its roof (c.f. Fig. 1). We then ran the combine control program. The combine robot searched for the fiducial marker as described in Section III. After the marker was detected, the spout was positioned according to the basic concept. A series of searching-positioning operations was autonomously performed by the developed software program.

Two types of positional relations between the combine robot and fiducial marker were tested. In case 1, the marker was located in an area where it could be captured by the primary searching step. In case 2 , the marker was located in an area where it could not be captured by the primary searching step but could be captured by the secondary searching step. We conducted the test three
50 times for each case and recorded the angular 51 displacement and required time for spout positioning.

For test 1 of case 1 and test 3 of case 2, the heights of the farm road $h_{f r}$ were 2.12 and $1.06 \mathrm{~m}$, respectively, whereas $h_{f r}$ was $0.00 \mathrm{~m}$ in the other tests. In addition, the position and orientation of the truck relative to the combine were the same for tests 2 and 3 of case 1 and tests 1 and 2 of case 2 .

Figure 7 shows the experimental results for (a1, a2) case 1 and (b1, b2) case 2. The grey dotted line shows the locus of the auger spout during the searching process, while the red circle indicates the positioning process. The red rectangle represents the estimated position of the fiducial marker. As illustrated in Figs. 7(a1) and (b1), the primary step started its path from the default position (i.e., $\theta_{1}=192.0^{\circ}$ and $\theta_{2}=2.6^{\circ}$; marked as $\mathrm{N} 1$ in the figure). The unloading auger raised its joint angle $\theta_{2}$ up to $45^{\circ}$ (marked as N2) and then rotated clockwise until it reached the next node N3. During this rotation, the fiducial marker was successfully detected at the spout position S1 $\left(\theta_{1}=37.5^{\circ}\right.$ and $\left.\theta_{2}=45^{\circ}\right)$. During the positioning, the auger rotated anticlockwise from S1 via S2 $\left(\theta_{1}=44.6^{\circ}\right.$ and $\left.\theta_{2}=45^{\circ}\right)$ to S3 $\left(\theta_{1}=44.6^{\circ}\right.$ and $\theta_{2}=$ $\left.29.8^{\circ}\right)$. In this instance, it took $7.6 \mathrm{~s}$ for the fiducial marker to be detected. After $2.2 \mathrm{~s}$ of rest to obtain clearer images, the robot started the spout positioning, which took $2.0 \mathrm{~s}$

Figs. 7(b1) and (b2) show the results when the camera found the fiducial marker in the second searching step. After the end of the primary searching step (N3: $\theta_{1}=0^{\circ}$ and $\theta_{2}=45^{\circ}$ ), the unloading auger lowered its joint angle $\theta_{2}$ down to $30^{\circ}$ (marked as $\mathrm{N} 4$ ) and then rotated anticlockwise to $\theta_{1}=90^{\circ}$. The fiducial marker was detected at the spout position $\mathrm{S} 4\left(\theta_{1}=54^{\circ}\right.$ and $\left.\theta_{2}=30^{\circ}\right)$. After a few seconds, the auger was positioned to $\theta_{1}=27^{\circ}$ and $\theta_{2}=0^{\circ}$ (marked as S5). The primary searching step took $9.6 \mathrm{~s}$, and it took $4.6 \mathrm{~s}$ from the end of the primary step for the fiducial marker to be detected. The positioning step took $3.6 \mathrm{~s}$ in this case. Overall, positioning took $23 \mathrm{~s}$.

Table 2 summarizes the time required for the searching-positioning operation; three tests were performed for each case. The table also contains $h_{f r}$ and the required angular displacement from the default position to the target position. Overall, $\theta_{2}$ (up) was constant because the unloading auger was only raised at the beginning of the searching procedure. 
1

\begin{tabular}{|c|c|c|c|c|c|c|}
\hline \multirow{2}{*}{ Case } & \multirow{2}{*}{ Test } & \multirow{2}{*}{$\begin{array}{c}\text { Required } \\
\text { time [s] }\end{array}$} & \multicolumn{3}{|c|}{ Angular displacement $\left[{ }^{\circ}\right]$} & \multirow{2}{*}{$\begin{array}{l}h_{f r} \\
{[\mathrm{~m}]}\end{array}$} \\
\hline & & & $\theta_{1}$ & $\theta_{2}$ (up) & $\theta_{2}($ down $)$ & \\
\hline \multirow{3}{*}{1} & 1 & 12 & 147.4 & 42.4 & 15.2 & 2.12 \\
\hline & 2 & 18 & 133.5 & 42.4 & 45.0 & 0.00 \\
\hline & 3 & 17 & 133.9 & 42.4 & 45.0 & 0.00 \\
\hline \multirow{3}{*}{2} & 1 & 23 & 218.6 & 42.4 & 45.0 & 0.00 \\
\hline & 2 & 22 & 216.8 & 42.4 & 45.0 & 0.00 \\
\hline & 3 & 16 & 205.9 & 42.4 & 30.6 & 1.06 \\
\hline
\end{tabular}

Manual operation

$37 \mathrm{~s}$ (on average)

9 For case 1 , test 1 clearly required less time. $h_{f r}$ was 10 higher than for the other two tests with this case, and the 11 target point was also higher. Thus, the angular 12 displacement of $\theta_{2}$ (down) was small. Compared to case 131 , case 2 took longer as a whole. This is because case 2 14 required a larger angular displacement of $\theta_{1}$. However, and (b2) top view of case 2 trajectory.
2

(a1)

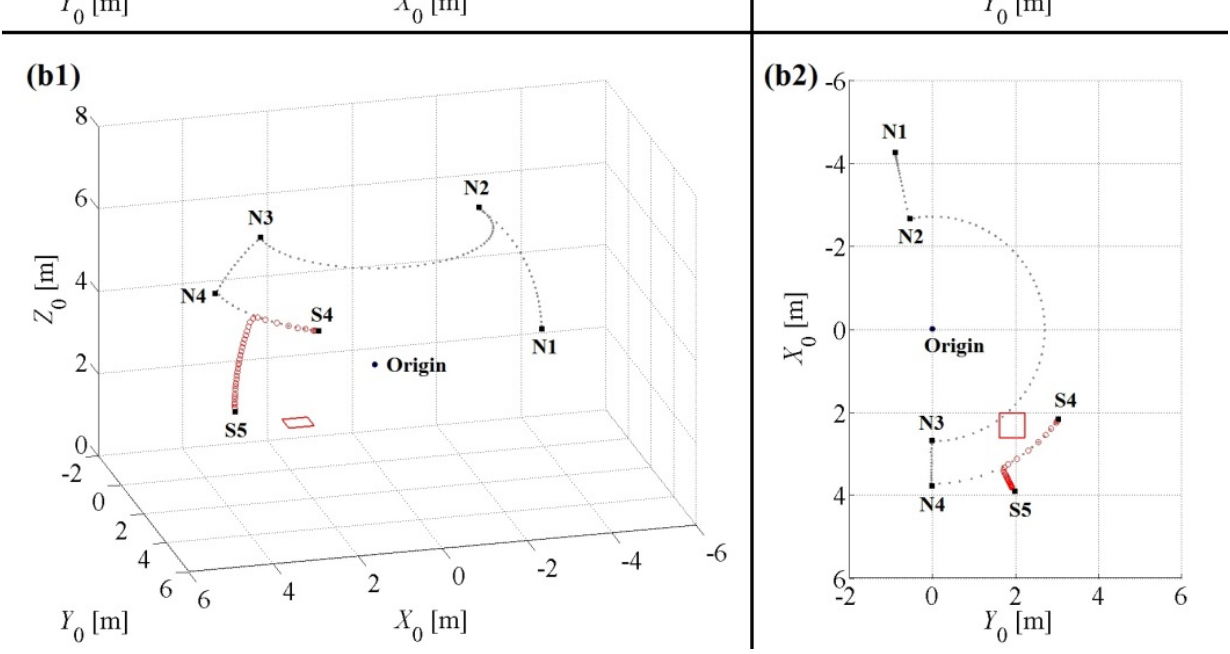

$($ a

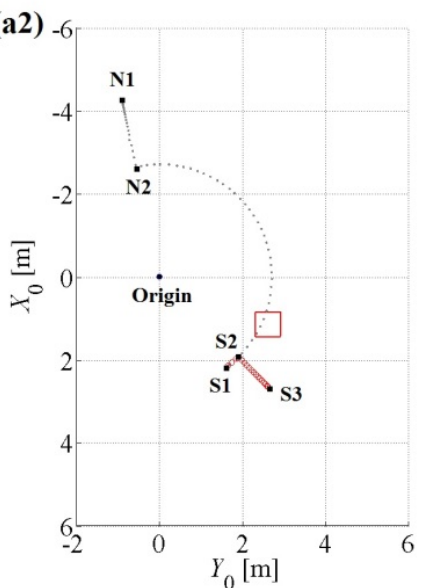

(b2)

Fig. 7. Trajectory of auger spout: (a1) overhead view and (a2) top view of case 1 trajectory; (b1) overhead view 


\section{Summary}

In order to realize efficient marker searching operation, 3 the FOV of an auger camera was computed on the basis of decision variables associated with the FOV. Under the assumption that the marker is searched for by the auger camera, we proposed an efficient marker searching procedure on the basis of the simulation results.

The maximal FOVs at representative $\theta_{2}$ values were 9 computed. The simulation results indicated that $92 \%$ of

Buckmaster, D. R. and Hilton, J. W. 2005. Computerized cycle analysis of harvest, transport, and unload systems. Computer and Electronics in Agriculture 47: 137-147.

Gonzalez, R. C. and Wintz, P. 1987. Digital Image Processing $2^{\text {nd }}$ Edition. Addison-Wesley Publishing Company, MA, USA.

Iida, M., Uchida, R., Zhu, H., Suguri, M., Kurita, H. and Masuda, R. 2013. Path-following control for a head-feeding combine robot. Engineering in Agriculture, Environment and Food 6(2): 61-67.

Ishida, M., Imou, K., Okado, A., Takenaga, H., Honda, Y., Itokawa, N. and Shibuya, Y. 1998. Autonomous tractor for forage production. Journal of the Japanese Society of
Agricultural Machinery 60(2): 59-66.

Kurita, H., Iida, M., Suguri, M. and Masuda, R. 2012. Application of Image Processing Technology for Unloading Automation of Robotic Head-Feeding Combine Harvester. Engineering in Agriculture, Environment and Food 5(4): 146-151.

Nagasaka, Y., Umeda, N., Kanetani, Y., Taniwaki, K. and Sasaki, Y. 2004. Automated rice transplanter using global positioning and gyroscopes. Computer and Electronics in Agriculture 43: 223-234.

Noguchi, N. and Terao, H. 1997. Path planning of an agricultural mobile robot by neural network and genetic algorithm. Computer and Electronics in Agriculture 18: 187-204.

Salaris, P., Pallottino, L., Hutchinson, S. and Bicchi, A. 2011. From Optimal Planning to Visual Servoing with Limited FOV. In Proc. 2011 IEEE/RSJ International Conference on Intelligent Robots and Systems, 2817-2824. San Francisco, CA., 25-30 September.

Takai, R., Barawid, O. Jr., Ishii, K. and Noguchi, N. 2010. Development of Crawler-Type Robot Tractor based on GPS and IMU. Preprint of the IFAC International Conference on AGRICONTROL 2010, A3-5. Kyoto, Japan, 6-8 December. Tsotsos, J. K. and Shubina, K. 2007. Attention and Visual Search: Active Robotic Vision Systems that Search. Keynote Lecture of the $5^{\text {th }}$ International Conference on Computer Vision Systems, Bielefeld, Germany, 21-24 May.

Xu, G. and Zhang, Z. 1996. Epipolar Geometry in Stereo, Motion and Object Recognition: A Unified Approach. Norwell: Kluwer Academic Publishers.

Zhang, H. and Ostrowski, J. P. 2002. Visual Motion Planning for Mobile Robots. IEEE Transactions on Robotics and Automation 18(2), 199-208. 